\title{
ON PROXIMATE ORDERS OF INTEGRAL FUNCTIONS
}

S. M. SHAH

Let $f(z)$ be an integral function of finite order $\rho$ and let $M(r)$ $=\max _{|z|-r}|f(z)|$. It is possible to find ${ }^{1}$ a positive continuous function $\rho(r)$ having the following properties.

(1) $\rho(r)$ is differentiable for $r>r_{0}$ except at isolated points at which $\rho^{\prime}(r-0)$ and $\rho^{\prime}(r+0)$ exist;

$$
\begin{array}{r}
\lim _{r \rightarrow \infty} \rho(r)=\rho ; \\
\lim _{r \rightarrow \infty} r \rho^{\prime}(r) \log r=0 ; \\
\limsup _{r \rightarrow \infty} \frac{\log M(r)}{r^{\rho(r)}}=1 .
\end{array}
$$

Such a function is called a Lindelö's proximate order for the integral function $f(z)$. The proof given by Valiron for the existence of proximate orders is based on some rather deep results due to Blumenthal. The object of this note is to give a particularly simple proof of the existence of proximate orders. The proof given here makes no use of the special properties of $M(r)$ and is therefore of wider scope.

Let $\sigma(r)=\log \log M(r) / \log r$. Either (A) $\sigma(r)>\rho$ for a sequence of values of $r$ tending to infinity, or (B) $\sigma(r) \leqq \rho$ for all large $r$.

In case (A) we define $\phi(r)=\max _{x \geqq r}\{\sigma(x)\}$. Since $\sigma(r)$ is continuous, $\lim \sup _{r \rightarrow \infty} \sigma(r)=\rho$ and $\sigma(r)>\rho$ for a sequence of values of $r$ tending to infinity. Therefore $\phi(r)$ exists. $\phi(r)$ is a nonincreasing function of $r$.

Let $r_{1}>e^{e^{\circ}}$ and $\phi\left(r_{1}\right)=\sigma\left(r_{1}\right)$. Such values will exist for a sequence of values of $r$ tending to infinity.

Let $\rho\left(r_{1}\right)=\phi\left(r_{1}\right)$. Let $t_{1}$ be the smallest integer not less than $1+r_{1}$ such that $\phi\left(r_{1}\right)>\phi\left(t_{1}\right)$, and let $\rho(r)=\rho\left(r_{1}\right)=\phi\left(r_{1}\right)$ for $r_{1}<r \leqq t_{1}$.

Define $u_{1}$ as follows:

$$
\begin{array}{rlrl}
u_{1} & >t_{1}, \\
\rho(r) & =\rho\left(r_{1}\right)-\log \log \log r+\log \log \log t_{1} & \text { for } t_{1} \leqq r \leqq u_{1}, \\
\rho(r) & =\phi(r) & \text { for } r & =u_{1},
\end{array}
$$

but $\rho(r)>\phi(r)$ for $t_{1} \leqq r<u_{1}$. Let $r_{2}$ be the smallest value of $r$ for which $r_{2} \geqq u_{1}$ and

Received by the editors November 10, 1945 .

${ }^{1} \mathrm{G}$. Valiron, Lectures on the general theory of integral functions, Cambridge, 1923, pp. 64-67. 


$$
\phi\left(r_{2}\right)=\sigma\left(r_{2}\right) .
$$

If $r_{2}>u_{1}$, then let $\rho(r)=\phi(r)$ for $u_{1} \leqq r \leqq r_{2}$. Since $\phi(r)$ is constant for $u_{1} \leqq r \leqq r_{2}$, therefore $\rho(r)$ is constant for $u_{1} \leqq r \leqq r_{2}$. We repeat the argument and obtain that $\rho(r)$ is differentiable in adjacent intervals. Further

$$
\rho^{\prime}(r)=0 \text { or } \frac{-1}{r \log r \log \log r},
$$

and $\rho(r) \geqq \phi(r) \geqq \sigma(r)$, for all $r \geqq r_{1}$. Further $\rho(r)=\sigma(r)$ for an infinity of values of $r=r_{1}, r_{2}, \cdots, \rho(r)$ is nonincreasing and $\lim _{r \rightarrow \infty} \phi(r)=\rho$. Hence

$$
\limsup _{r \rightarrow \infty} \rho(r)=\lim _{r \rightarrow \infty} \rho(r)=\rho,
$$

and since $\log M(r)=r^{\sigma(r)}=r^{\rho(r)}$ for an infinity of $r, \log M(r)<r^{\rho(r)}$ for the remaining $r$, therefore $\lim \sup _{r \rightarrow \infty} \log M(r) / r^{\rho(r)}=1$.

Let $\sigma(r) \leqq \rho$ for all large $r$ (case (B)). Here there are two possibilities:

$$
\sigma(r)=\rho
$$

for at least a sequence of values of $r$ tending to infinity;

$$
\sigma(r)<\rho
$$

for all large values of $r$.

In case (B.1) we take $\rho(r)=\rho$ for all values of $r$.

In case (B.2) let $\xi(r)=\max _{X \leqq x \leqq r}\{\sigma(x)\}$ where $X>e^{\theta^{\circ}}$ is such that $\sigma(x)<\rho$ whenever $x \geqq X . \xi(r)$ is nondecreasing. Take a suitably large value of $r_{1}>X$ and let

$$
\begin{aligned}
\rho\left(r_{1}\right) & =\rho, \\
\rho(r) & =\rho+\log \log \log r-\log \log \log r_{1}
\end{aligned}
$$$$
\text { for } s_{1} \leqq r \leqq r_{1},
$$

where $s_{1}<r_{1}$ is such that $\xi\left(s_{1}\right)=\rho\left(s_{1}\right)$. If $\xi\left(s_{1}\right) \neq \sigma\left(s_{1}\right)$, then we take $\rho(r)=\xi(r)$ up to the nearest point $t_{1}<s_{1}$ at which $\xi\left(t_{1}\right)=\sigma\left(t_{1}\right) . \rho(r)$ is then constant for $t_{1} \leqq r \leqq s_{1}$.

If $\xi\left(s_{1}\right)=\sigma\left(s_{1}\right)$, then let $t_{1}=s_{1}$.

Choose $r_{2}>r_{1}$ suitably large and let $\rho\left(r_{2}\right)=\rho$,

$$
\rho(r)=\rho+\log \log \log r-\log \log \log r_{2} \quad \text { for } s_{2} \leqq r \leqq r_{2}
$$

where $s_{2}\left(<r_{2}\right)$ is such that ${ }^{2} \xi\left(s_{2}\right)=\rho\left(s_{2}\right)$. If $\xi\left(s_{2}\right) \neq \sigma\left(s_{2}\right)$, then let

\footnotetext{
${ }^{2} s_{2}$ is given by the largest positive root of $\xi\left(s_{2}\right)=\rho-\log \log \log r_{2}+\log \log \log s_{2}$.
} 
$\rho(r)=\xi(r)$ for $t_{2} \leqq r \leqq s_{2}$ where $t_{2}\left(<s_{2}\right)$ is the point nearest to $s_{2}$ at which $\xi\left(t_{2}\right)=\sigma\left(t_{2}\right)$. If $\xi\left(s_{2}\right)=\sigma\left(s_{2}\right)$, then let $t_{2}=s_{2}$. For $r<t_{2}$ let $\rho(r)$ $=\rho\left(t_{2}\right)+\log \log \log t_{2}-\log \log \log r$ for $u_{1} \leqq r \leqq t_{2}$ where $u_{1}\left(<t_{2}\right)$ is the point of intersection of $y=\rho$ with $y=\rho\left(t_{2}\right)+\log \log \log t_{2}-\log \log \log r$.

Let $\rho(r)=\rho$ for $r_{1} \leqq r \leqq u_{1}$. It is always possible to choose $r_{2}$ so large that $r_{1}<u_{1}$. We repeat the procedure and note that

$$
\rho(r) \geqq \xi(r) \geqq \sigma(r)
$$

and $\rho(r)=\sigma(r)$ for $r=t_{1}, t_{2}, t_{3}, \cdots$. Hence $\lim _{r \rightarrow \infty} \rho(r)=\rho$, and

$$
\limsup _{r \rightarrow \infty} \frac{\log M(r)}{r^{\rho(r)}}=1 \text {. }
$$

MUSLIM UNIVERSITY

\section{A NOTE ON THE SPECTRAL THEOREM}

\section{WILLIAM F. EBERLEIN}

1. Introduction. Although the connections between the spectral resolution of a self-adjoint transformation in Hilbert space, the moment problem, and Riesz' integral representation [1] ${ }^{1}$ for linear functionals on the space $C$ are known (cf. Stone [2], Murray [3], Widder [4], Lengyel [5]), the following elementary derivation of the spectral theorem from the Riesz theorem exhibits the connections in, perhaps, the simplest light. We consider only bounded self-adjoint transformations $H$; one can treat an unbounded $H$ by considering $\left(I+H^{2}\right)^{-1}$, which is bounded and self-adjoint $[3$, p. 95]. Note that the derivation does not involve the separability of the Hilbert space $\mathfrak{W}$.

2. Six lemmas. Let $H$ be a self-adjoint transformation with the bounds $a, b$ - that is, $a\|f\|^{2} \leqq(H f, f) \leqq b\|f\|^{2}$ for all $f \in H$, and $\|H\|$ $=\max (|a|,|b|)$. Denote by $C$ the space of continuous real-valued functions defined on the closed interval $(a, b)$, with $\|f(x)\|=\max |f(x)|$ $(a \leqq x \leqq b)$. Let $p(x)=\sum_{0}^{n} c_{j} x^{i}$ be any polynomial with real coefficients, and let $p(H)$ be the corresponding transformation $p(H)=\sum_{0}^{n} c_{j} H^{i}$, where $H^{0}=I$.

Received by the editors September 6, 1945.

${ }^{1}$ Numbers in brackets refer to the references cited at the end of the paper. 